\title{
Procarcinogenic effect of Ginkgo biloba L. extract in a hepatocarcinogenesis model
}

\author{
Héctor Donaldo Zenón-Alanco ${ }^{1}$, José Locia-Espinoza ${ }^{2}$, Arturo Venebra-Muñóz ${ }^{3}$ and Luz Irene \\ Pascual-Mathey ${ }^{4}$
}

${ }^{1}$ Facultad de Bioanálisis, Universidad Veracruzana, Xalapa, Ver.

${ }^{2}$ Facultad de Química Farmacéutica Biológica, Universidad Veracruzana, Xalapa, Ver.

${ }^{3}$ Facultad de Medicina, Universidad Autónoma del Estado de México, Toluca, Méx.

${ }^{4}$ Facultad de Química Farmacéutica Biológica, Universidad Veracruzana, Xalapa, Ver.

*Corresponding author: Luz I Pascual-Mathey, Facultad de Química Farmacéutica Biológica, Circuito Gonzalo Aguirre Beltrán s/n, Zona Universitaria, Xalapa, Veracruz, 91190, Mexico.

To Cite This Article: Héctor Donaldo Zenón-Alanco, José Locia-Espinoza, Arturo Venebra-Muñóz, Luz Irene Pascual-Mathey. Procarcinogenic effect of Ginkgo biloba L. extract in a hepatocarcinogenesis model. Am J Biomed Sci \& Res. 2021 - 13(1). AJBSR.MS.ID.001839.

DOI: 10.34297/AJBSR.2021.13.001839.

Received: 䟧 June 02, 2021; Published: 制 June 09, 2021

\begin{abstract}
Currently, hepatocellular carcinoma (HCC) is one of the leading causes of death worldwide. Among the treatment alternatives are phytomedicines. Ginkgo biloba extract (EGb) is a herbal medicine with antioxidant and antiproliferative properties reported in different "in vitro" models of cancer, so, it could be used to treat HCC. However, recent studies show that it can also have carcinogenic effects. Therefore, through the quantification of the enzymes ALT and AST, serum markers of hepatocellular damage, the impact of EGb was evaluated in a model of hepatocarcinogenesis in the initiation and promotion stages in male rats of the Wistar strain. For this, 21 two-month-old Wistar male rats were used, separated into seven groups. The administration of DEN induced hepatocarcinogenesis for the initiation stage and 2-AAF for the promotion stage. Subjects underwent a 96-hour fast before DEN administration to induce cell proliferation. Group 1 was not administered with any treatment (intact); group 2 received only the vehicle (control); from the third to the seventh group, they were issued with the carcinogens DEN (day 0; initiation) and 2-AAF (6 doses, one every third day from day 14; promotion). Groups 4 and 5 received 160 and $500 \mathrm{mg} / \mathrm{kg}$ of EGb from day one until the completion of treatment, while groups 6 and 7 were administered with 160 and $500 \mathrm{mg} / \mathrm{kg}$ of EGb from day 14 until the completion of the model. At the end of the treatment (day 40), blood was obtained, and the ALT and AST enzymes' values were quantified.
\end{abstract}

Keywords: Ginkgo biloba, Phytotherapy, Carcinogenic, Hepatocellular Cancer, Biomarkers, Transaminases, ALT, AST.

\section{Introduction}

HCC is considered one of the leading causes of death globally, being the third type of cancer with the highest mortality (more than one million deaths per year) [1].

Current treatments for HCC have proven to be inadequate because they risk generating undesirable effects, being an example of these medications, corticosteroids, and interferon, in addition to the fact that they are costly. Therefore, treatments based on natural compounds have been used as an accessible alternative that can contribute to the treatment of this disease [2].
Ginkgo biloba (EGb) extract is recognized for being rich in antioxidants and reducing oxidative stress, inducing cytotoxicity and apoptosis, and inhibiting cell proliferation in "in vitro" models of breast and pancreatic cancer cell lines, among others [3-4]. However, recent studies have shown that it can increase the incidence of different types of cancer, including HCC [5].

Hepatocarcinogenesis is induced through different "in vivo" models that combine the use of substances such as Diethylnitrosamine (DEN) and 2-Acetylaminofluorene (2-AAF), in 
conjunction with the use of mitotic stimuli (such as fasting and liver resection) [ 6-7]. In addition, some markers evaluate liver damage, among which are the enzymes alanine aminotransferase (ALT) and aspartate aminotransferase (AST), which are considered prognostic indicators to estimate HCC because their increase is associated with the destruction of hepatocytes due to tumor growth, circulatory alterations due to portal vein thrombosis, cirrhosis, alcoholic liver diseases, etc. [8].

Therefore, the objective of this study was to evaluate the levels of ALT and AST in a model of hepatocarcinogenesis induced by the administration of DEN and 2-AAF joint with EGb in the stages of initiation and promotion of hepatocarcinogenesis.

\section{Matherials and methods}

Twenty-one 2-month-old Wistar male rats were housed under ad libitum conditions of water and food with light-dark periods of 12 hours. The care of the animals was carried out based on the Official Mexican Standard NOM-062-ZOO-1999. Carcinogenesis was induced according to the protocol of Espandiari et al. (2005) with certain modifications. Diethylnitrosamine (DEN; Sigma-Aldrich), was used for initiation and 2-acetylaminofluorene (2-AAF; SigmaAldrich) for the promotion stage. As a proliferative mitotic stimulus [9], a 96-hour fast was used before the administration of DEN (200 $\mathrm{mg} / \mathrm{kg}$, day 0) dissolved in propylene glycol by the intraperitoneal route. Two weeks after DEN administration, subjects received an oral dose of 2-AAF (20 mg/kg in propylene glycol) for a total of 6 doses (days 14, 16, 18, 20,22, and 24). Groups 4 and 5 were administered with 160 and $500 \mathrm{mg} / \mathrm{kg}$ of EGb (Vasodil ${ }^{\circledR}$ ) from day one until the completion of treatment, while groups 6 and 7 were administered with 160 and $500 \mathrm{mg} / \mathrm{kg}$ of EGb from day 15 until the completion of the model. At the end of the treatment (40 days), blood was extracted by renal puncture, and quantification of the ALT and AST enzymes (SPINREACT ${ }^{\circledR}$ ) was asaayed, with a UVVIS 6300 JENWAY spectrophotometer. The results were analyzed using a one-way ANOVA with the SigmaStat 3.5 program, showing the mean \pm SE when the significant differences were greater than $\mathrm{P}<0.05$, using the post hoc Student Newman Keuls test to evaluate the statistical differences between groups.

\section{Results}

The analysis of the biomarkers of liver damage showed significant differences between the different treatments $(\mathrm{P}<0.01)$. When analyzing the enzyme Aspartate aminotransferase (AST or GOT), an increase was observed in the groups with the administration of Ginkgo biloba extract both in the initiation and promotion stages with the doses of 160 and $500 \mathrm{mg} / \mathrm{kg}$ (G4-G7), in contrast to the results shown in the intact and control groups. Concerning Alanine aminotransferase (ALT or GPT), an enzyme located mainly in the cytosol of hepatocytes, the post hoc test showed an increase in serum levels in groups with cancer and administration of Ginkgo biloba extract in both stages (initiation and promotion) with the doses of 160 and $500 \mathrm{mg} / \mathrm{kg}$ (G3-G7), without this increase being significant in group 7 (Table 1).

Interestingly, in the group that only received DEN and 2-AAF carcinogens administration, the increase of both enzymes (AST and ALT) was not significant, as observed in the groups that received administration of EGb (Table 1).

Table 1: AST and ALT levels in serum of subjects with DEN, 2-AAF and EGb administration.

\begin{tabular}{|c|c|c|c|c|c|c|c|}
\hline & G1 & G2 & G3 & G4 & G5 & G6 & G7 \\
\hline AST & $39.08 \pm 2.20$ & $45.20 \pm 3.38$ & $48.41 \pm 3.03$ & $75.83 \pm 3.48^{* *}$ & $80.20 \pm 8.42^{* *}$ & $63.00 \pm 6.02^{*}$ & $53.66 \pm 3.48$ \\
\hline ALT & $18.32 \pm 0.58$ & $20.41 \pm 2.06$ & $26.83 \pm 2.69$ & $31.11 \pm 3.20^{*}$ & $29.75 \pm 3.54^{*}$ & $34.51 \pm 3.03^{*}$ & $28.48 \pm 1.51$ \\
\hline
\end{tabular}

ALT $=$ Alanine aminotransferase; AST $=$ Aspartate aminotransferase. Differences are presented with relation to the intact group $\left(p<0.05^{*}, p<0.01^{\star}\right)$.

Data represent mean $\pm \mathrm{EE}$.

\section{Discussion}

EGb has been a widely used herbal medicine for the treatment/ prevention of many diseases, including cancer [3-4]. In the results of this study, in which HCC was induced through the administration of the carcinogens DEN and -AAF, a significant increase in the levels of ALT and AST transaminases is shown in the groups that received $\mathrm{EGb}$ administration in comparison with the groups that only received administration of the carcinogens. The increase in ALT has been found in more significant amounts in kidney and heart diseases, specifically in the liver, than other tissues, so any damage to this organ could cause an increase in this enzyme. On the other hand, although AST transaminase is less specific than ALT, its increase has been reported in chronic liver diseases related to liver tissue degeneration and necrotizing processes [10]. Furthermore, its elevation has been reported in patients with cirrhosis and liver diseases where the main elevated enzyme is ALT. On the other hand, the AST/ALT ratio increase has been related to an imbalance of liver function. Specifically, it has been shown that a higher ratio of AST to ALT occurs in alcoholic liver disease, hepatitis, fibrosis, chronic infections, etc. [10]. The study carried out by Changchien et al. (2008), which evaluated the levels of transaminases in a population of 6381 patients with HCC, showed that the increase in 
these transaminases is associated with hepatitis and destruction of hepatocytes due to tumor growth. In addition, the increase in the AST/ALT ratio indicates a poor prognosis since it is associated with an increase in tumor size. Therefore, the results found in this study, where an increase in both transaminases is observed, with an AST >ALT ratio, suggests that the administration of EGb could be causing liver damage greater than that of carcinogenic compounds, favoring the development of carcinogenesis, as previously reported [5], which requires further investigation, so, should take its consumption with caution.

\section{Acknowledgments}

To the National Council of Science and Technology (CONACyT).

\section{References}

1. Lombardi A, Grimaldi A, Zappavigna S, Misso G, Caraglia M (2018) Hepatocarcinoma: genetic and epigenetic features. Minerva Gastroenterologica e Dietologica. 64(1): 14-27.

2. Ramos-Tovar E, Muriel P (2019) Phytotherapy for the Liver. Dietary Interventions in Liver Disease. 101-121.

3. Park YJ, Kim MJ, Kim HR, Yi MS, Chung KH, et al. (2013) Chemopreventive effects of Ginkgo biloba extract in estrogen-negative human breast cancer cells. Arch Pharm Res 36(1): 102-108.
4. Zhang Y, Chen A, Li M, Chen C, Yao Q (2008) Ginkgo biloba Extract Kaempferol Inhibits Cell Proliferation and Induces Apoptosis in Pancreatic Cancer Cells, Journal of Surgical Research, 148 (1): 17-23.

5. Kovi RC, Bhusari S, Mav D, Shah RR, Ton TV, Hoenerhoff MJ, et al. (2019) Genome-wide promoter DNA methylation profiling of hepatocellular carcinomas arising either spontaneously or due to chronic exposure to Ginkgo biloba extract (GBE) in B6C3F1/N mice. Arch Toxicol 93(8): 2219-2235.

6. Espandiari P, Robertson LW, Srinivasan C, Glauert HP (2005) Comparison of different initiation protocols in the resistant hepatocyte model. Toxicology. 206(3): 373-381.

7. Santos NP, Colaço AA, Oliveira PA (2017) Animal models as a tool in hepatocellular carcinoma research: A Review. Tumour Biol. 39(3):1010428317695923.

8. Changchien CS, Chen CL, Yen YH, Wang JH, Hu TH, et al. (2008) Analysis of 6381 hepatocellular carcinoma patients in southern Taiwan: prognostic features, treatment outcome, and survival. Journal of gastroenterology 43(2): 159-170.

9. Amin A, Daoud S, Zaki N, Salehi-Ashtiani K, Al-Hrout A, Chaiboonchoe A, Baig B, Khazanehdari K, \& Adrian T (2016) Saffron-based crocin prevents early lesions of liver cancer: In vivo, in vitro and in silico analyses. Hpb 18.

10. Gowda S, Desai PB, Hull VV, Math AA, Vernekar SN, et al. (2009) A review on laboratory liver function tests. The Pan African medical journal 3: 17. 